\title{
Place learning in the Morris place navigation task is impaired by damage to the hippocampal formation even if the temporal demands are reduced
}

\author{
ROBERT J. SUTHERLAND \\ University of Lethbridge, Lethbridge, Alberta, Canada \\ and \\ JERRY W. RUDY \\ University of Colorado, Boulder, Colorado
}

\begin{abstract}
Rats with hippocampal-formation damage display a persisting impairment of place learning in the Morris water task. In this paper, we evaluated the temporary memory buffer acount of hippocampal function (Rawlins, 1985, 1987) as an explanation for this place-learning impairment. The essence of this view is that the Morris task requires the animal to integrate information about its position and the relationships among the goal and distal cues over a long temporal interval. Animals with damage to the hippocampal formation are impaired because the temporal demands of the task exceed what can be bridged in the absence of an intact hippocampal formation. To evaluate this hypothesis, we reduced the temporal demands of the Morris task to a minimum by training animals to swim directly to a single, visible platform in a fixed location and subsequently probing them with trials using a hidden platform in the same location. In two experiments based on this general design, animals with colchicine-induced damage to the hippocampal formation were significantly impaired relative to control animals. Thus, our data provide no support for the temporary memory buffer account.
\end{abstract}

The place learning version of the Morris (1981) water task requires animals to locate an escape platform that is hidden beneath the surface of a pool of water. Because the location of the hidden platform is fixed in relation to extramaze cues, this task is generally thought to be a member of a class whose normal solution depends upon the use of what O'Keefe and Nadel (1978) have called a locale strategy. The distinguishing feature of a locale strategy is that it permits the animal to move directly to a goal object from a variety of different starting locations by learning the location of the goal relative to more distal cues in the environment. Rats that solve this problem are said to have true allocentric spatial mapping ability. O'Keefe and Nadel propose that the hippocampal formation provides the neural basis for learning and remembering spatial maps.

Rats with damage to the hippocampal formation or entorhinal cortex are severely impaired in the hidden-

\footnotetext{
This research was supported in part by grants to $R$. J. Sutherland from the Natural Science and Engineering Research Council of Canada and to J. W. Rudy from the National Science Foundation (Grant BNS8207654). Correspondence concerning this article can be sent to either R. J. Sutherland, Department of Psychology, The University of Lethbridge, 4401 University Drive, Lethbridge, Alberta T13M4, Canada, or Jerry W. Rudy, Department of Psychology, University of Colorado, Boulder, CO 80309.
}

platform, fixed-location version of the Morris (1981) water task (Morris, Garrud, Rawlins, \& O'Keefe, 1982; Schenk \& Morris, 1985; Sutherland, 1985; Sutherland, Kolb, \& Whishaw, 1982; Sutherland, Whishaw, \& Kolb, 1983). This finding offers strong support for O'Keefe and Nadel's (1978) spatial-map theory of hippocampalformation function.

Recently, however, Morris, Hagan, and Rawlins (1986) presented data obtained from a variation of the Morris (1981) water task that they interpreted as being problematic for spatial-mapping theory. Specifically, Morris et al. (1986) trained rats to discriminate between two visible, nominally identical platforms. Only one platform provided an escape from the water. It was rigid and would support the rats' weight. The other platform did not provide an escape. It floated but would sink when the rats attempted to climb onto it. The rigid platform was always in a fixed location relative to the extramaze cues, whereas the floating-platform location varied from trial to trial. We will refer to this task as the cued placediscrimination task. Most of the rats with damage to the hippocampal formation were severely impaired relative to controls in learning to solve this spatial discrimination. Nevertheless, 4 of the 10 animals with hippocampalformation damage did eventually reach a criterion of $85 \%$ correct. According to Morris et al. (1986), these results are problematic for O'Keefe and Nadel's (1978) position, 
because spatial-map theory predicts an absolute deficit in this task.

We are not impressed that Morris et al.'s (1986) data are especially problematic for spatial-map theory. The primary concern of our paper, however, is Morris et al.'s (1986) additional claim that their results provide support for Rawlins's (1985) theory of hippocampal-formation function. Briefly, Rawlins proposed that the hippocampal formation provided the neural substrate for a highcapacity, intermediate-term, temporary memory buffer, which would enable animals to form associations among temporally discontiguous events. According to Rawlins, successful place learning requires that the animal integrate information about its starting location, its route in transit, and the goal location in relation to distal cues viewed over an extended interval of time. The importance of a temporary memory buffer for place navigation in the Morris water task is seen by contrasting the hidden, fixedplatform task with the cued place-discrimination task used by Morris et al. (1986). Typically, during the initial trials of the hidden-platform task, even normal animals will swim for $30 \mathrm{sec}$ or more before finding the escape platform. Normal animals possess the necessary memory buffer for integrating the temporally discontiguous information needed to relate the different views to the extramaze cues seen from the starting location to the end of the swim at the hidden-platform location. Animals with damage to the hippocampal formation are absolutely impaired on the hidden-platform task because, according to Rawlins's (1985) position, they lack the temporary memory buffer needed to overcome the temporal demands of the task.

Animals with damage to the hippocampal formation are less impaired in the cued place-discrimination task than in the hidden-platform task because hippocampal damage does not impair the animals' ability to swim quickly and directly to a visible platform (Morris et al., 1982). Consequently, in the cued place-discrimination task, rats often locate the correct platform in 2-5 sec. Thus, the temporal interval between the start and finish of a swim is greatly reduced relative to what is typically observed in the hidden-platform task, and the performance of animals with hippocampal-formation damage correspondingly improves.

Rawlins's (1985) theory may provide a plausible interpretation of the contribution of the hippocampal formation to place learning, but we are not at all convinced that the data Morris et al. (1986) offer should be considered as support for his theory. A primary problem is that the authors failed to provide any evidence that even the animals with hippocampal-formation damage who reached criterion on the cued place-discrimination task had learned the spatial location of the rigid, visible platform. Morris et al. (1986), for example, acknowledge the possibility that "uncontrolled 'Iocal' cues within the pool (e.g., waves reflected in different ways off the two platforms) might have served to guide discriminative performance" (p. 375). They claimed to offer evidence against this in- terpretation by demonstrating that the control animals' choice accuracy decreased systematically when the availability of extramaze relational cues was reduced. It is the spatial-mapping ability of the animals with hippocampalformation damage that is at issue, however, and Morris et al. (1986) inexplicably reported no data for how these animals performed when extramaze cues were removed. Without this information, it is gratuitous for the authors to advance the temporary memory buffer account at the expense of the spatial-mapping interpretation.

We attempted to provide a more direct test of Rawlins's (1985) theory by taking advantage of another phenomenon reported by Morris (1981). In one of his experiments, rats were trained to swim to a visible platform in a fixed location and then, after removal of the visible platform from the pool, were probed for their knowledge of the platform's location. Morris (1981) found that normal rats will search for the platform in the correct location within the pool even if they were trained with a visible platform.

\section{EXPERIMENT 1}

The use of a visible platform vastly reduces the temporal demands of the Morris water task and accordingly, if Rawlins's interpretation of the place navigation deficit is correct, should increase the likelihood that animals with damage to the hippocampal formation will learn the platform location in relation to distal cues (Morris et al., 1986; Rawlins, 1985, 1987). In our first experiment, we trained both control rats and rats with colchicine-induced damage to the hippocampal formation to navigate to a visible platform until their escape latencies had stabilized at about $2.5 \mathrm{sec}$. Then, on a series of probe trials, the visible platform was replaced by a hidden platform. These probe trials provided these animals with the opportunity to demonstrate knowledge of the platform location unconfounded by the presence in the pool of contaminating local cues.

\section{Method}

Subjects. The subjects were $13400-500-\mathrm{g}$ male hooded rats obtained from the University of Colorado colony. They were housed in individual cages and were provided with ad-lib access to food and water. The lights were on from 0700 to $1900 \mathrm{~h}$. Testing was carried out between 1300 and $1500 \mathrm{~h}$.

Apparatus. The animals were trained and tested in a large circular pool, $1.2 \mathrm{~m}$ in diameter and $.36 \mathrm{~m}$ deep. The water level in the pool was $.2 \mathrm{~m}$. Water temperature was maintained at $22^{\circ} \mathrm{C}$. The interior wall of the pool was white. The pool was located in a room that was $2.3 \times 2.73 \times 2.5 \mathrm{~m}$ high. The details of the room are fully described in an article by Rudy, Stadler-Morris, and Albert (1987).

The visible platform was $12 \times 12 \mathrm{~cm}$ and had black sides and a white top. It was made of wood and protruded $2.5 \mathrm{~cm}$ above the surface of the water. Hard wire screening was attached to the platform to facilitate the rat's climb to the platform top. The hidden platform was a clear Plexiglas circular disk, $12 \mathrm{~cm}$ in diameter. It was placed $1.5 \mathrm{~cm}$ below the surface of the water. To insure that the platform could not be seen, the water was rendered opaque by the addition of powdered white Crayola water paint. 
Surgery. Two weeks prior to training, the rats were randomly assigned to two groups. The animals in one group $(n=7)$ received intrahippocampal injections of colchicine while anesthetized with sodium pentobarbital $(60 \mathrm{mg} / \mathrm{kg}$, i.p.). The colchicine injections were stereotaxically placed and made through a 30 -ga stainless steel needle connected to a $10-\mu \mathrm{l}$ syringe (Hamilton) fixed to a motorized microdrive. The colchicine was dissolved in physiological saline $(4 \mathrm{mg} / \mathrm{ml})$. Over a $4-\mathrm{min}$ interval, $0.5 \mu \mathrm{l}$ was infused into each injection site. Injections were made at three sites within each hippocampus with the skull leveled between the bregma and lambda (coordinates 3.5 P, 1.4 L, 3.8 V; 5.0 P, 3.3 L, 3.8 V; 6.2 P, $4.6 \mathrm{~L}, 7.7 \mathrm{~V})$. The animals in the control group $(n=6)$ were treated identically except that the needle was not allowed to penetrate into the hippocampus (the ventral coordinate was $1.5 \mathrm{~mm}$ below the dura) and no drug was infused into the brain.

Histology procedure. After the completion of behavioral testing, the animals were deeply anesthesized with sodium pentobarbital (100 mg/kg, i.p.) and intracardially perfused with physiological saline follwed by $10 \%$ formal saline. The brains were stored in the formal saline solution; $\mathbf{4 8} \mathrm{h}$ prior to sectioning, they were transferred to a $10 \%$ formal saline, $30 \%$ sucrose solution. The brains were sectioned at $40 \mu \mathrm{m}$, and every 7 th section through the hippocampal formation was mounted on microscope slides and stained with cresyl violet for microscopic verification of the lesion.

Behavioral Testing. All rats were initially trained to swim to the visible platform, which was placed in the center of the NE quadrant of the pool. In training, each rat was given 4 trials per day. Each trial began when the rat was released from one of four starting locations and ended when the rat found the platform or when $90 / \mathrm{sec}$ had elapsed. If, after $90 / \mathrm{sec}$, the rat was still swimming, it was placed by hand onto the platform. The rat remained on the platform for $8 \mathrm{sec}$, after which it was returned to its holding cage. The intertrial interval was approximately $2 \mathrm{~min}$. Following every 12 training trials with the visible platform, each subject received a session of 4 probe trials in which the visible platform was replaced by the hidden platform. This sequence of training and probe trials continued until 36 training trials and 12 probe trials had been administered.

Dependent variables. On acquisition and probe trials, two measures of the animals' performance were analyzed: (1) escape latency, the time taken by the rats to find the platform, and (2) swim distance, the distance traveled before the rats found the platform. Since these two measures yielded the same result (i.e., the animals swam at very similar speeds), only the escape-latency data are presented.

\section{Results and Discussion}

Histology. The extent of damage was very similar to that found in previous investigations using the same microinjection procedures (Sutherland, 1985; Sutherland et al., 1982; Sutherland et al., 1983). The colchicine injections produced a massive loss of cells from the dorsal and ventral blades of the dentate gyrus throughout the septotemporal extent of the hippocampal formation (Figure 1). To a lesser extent, there was thinning of the CA1 subfield and the overlying neocortex around the tracts of the injection needles. In all rats, there were some granule cells spared; this was particularly noticeable in the extreme temporal part of the dentate gyrus.

Behavior. Figure 2 presents the escape latencies for the two groups of subjects averaged over the four daily trials with the visible platform and over the three blocks of four probe trials with the hidden platform. On Day 1 of training with the visible platform, the escape latencies of animals with damage to the hippocampal formation were long relative to those of the control animals. Thereafter, the two groups did not differ in finding the visible platform and had escape latencies that averaged less than $3 \mathrm{sec}$. On the first probe trial, the escape latencies of both groups increased significantly relative to baseline performance on trials with the visible platform, but did not differ from each other. Thereafter, the performance of the two groups on the probe trials differed sharply. The escape latencies of the animals with damage to the hippocampal formation not only continued to be longer than those they had established on trials with the visible platform, but now were also longer than those of the control animals. On the final two sets of trials with the hidden platform, the probe trial latencies for the control subjects did not differ significantly from their own escape latencies on trials with the visible platform.

These results were analyzed statistically by performing a two-way, repeated measures analysis of variance on the acquisition and probe data (averaged over the daily four trials). It revealed significant main effects of lesion $[F(1,12)=6.08, p<.05]$ and trials $[F(11,121)=8.77$, $p<.01]$ and a significant lesion $\times$ trials interaction $[F(11,121)=3.55, p<.01]$. Protected $t$ tests then revealed that (1) the escape latencies of the two groups were significantly different on both the second and third set of probe trials $(p<.01),(2)$ the escape latencies of the animals with damage to the hippocampal formation were significantly longer on each of the three blocks of probe trials than they were on the preceding blocks of trials with the visible platform ( $p s<.001$ ), and (3) it was only on the first block of probe trials that the escape latencies of the control animals were significantly longer $(p<.001)$ than they had been on the preceding block of trials with the visible platform.

This pattern of data provides no support for Rawlins's (1985) theory. Even though the animals with damage to the hippocampal formation had been swimming directly to the visible platform with escape latencies of less than $3 \mathrm{sec}$, their performance deteriorated sharply when they were required to find the hidden platform located in the same spot in the pool. In contrast, by the end of training, the control animals were finding the hidden platform almost as readily as they had located the visible platform. Thus, even though the temporal demands of the place navigation task were greatly reduced, animals with damage to the hippocampal formation were still clearly impaired.

Rats with damage to the hippocampal formation were initially impaired on the visible platform task. However, after only a few trials, both groups were navigating directly to the visible platform. The reason for the initial impairment in the lesion group is not obvious, but a similar transient impairment has been reported previously by Morris (1982, Figure 3). Our rats had received no experience in the task prior to visible cue training, and the lesioned rats' initial impairment may reflect a greater persistence in their searches along the wall of the pool. 

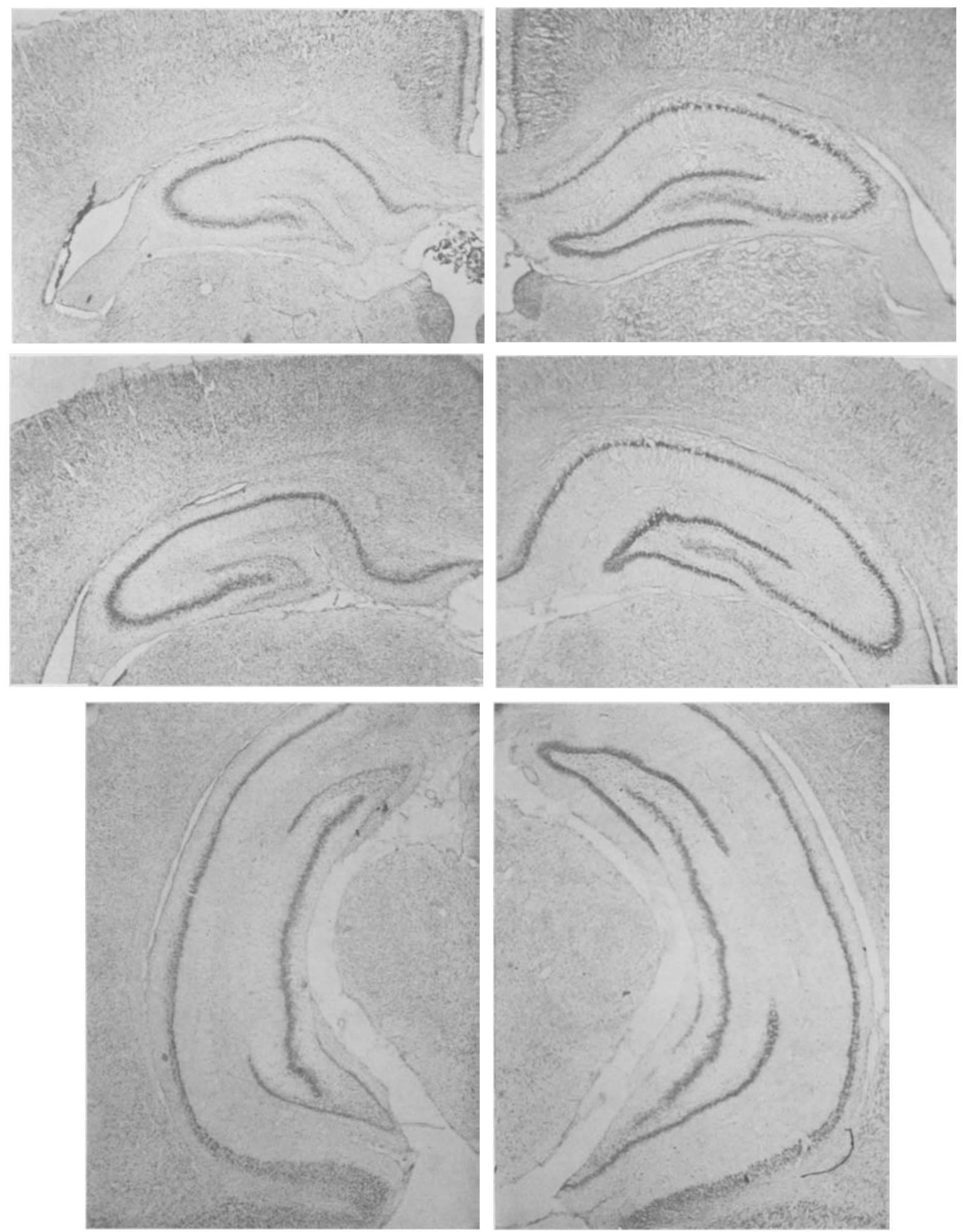

Figure 1. Left panel: Photomicrographs of coronal sections at three planes through the hippocampal formation from a typical colchicinetreated rat. Right panel: Photomicrographs of coronal sections through the hippocampal formation from a control rat. 


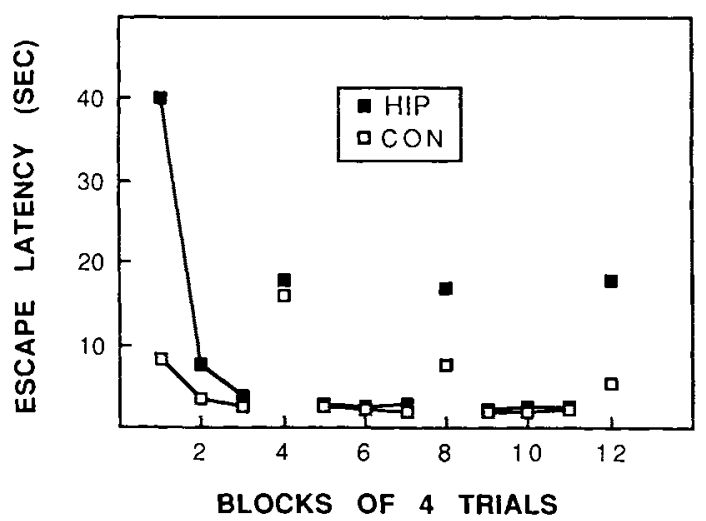

Figure 2. The mean escape latencies averaged over blocks of four trials with the visible platform (connected points) and hidden platform (unconnected points). Note that by the end of training, control animals were locating the hidden platform as rapidly as they were locating the visible platform. In contrast, the performance of animals with hippocampal-formation damage remained comparatively poor on trials with the hidden platform.

\section{EXPERIMENT 2}

Since the difference between hippocampal-damaged and control animals was not present on the initial block of probe trials but emerged only on the second and third blocks, one might argue that neither the controls nor the animals with hippocampal damage learned anything about the location of the platform on trials with the visible platform. The shorter escape latencies displayed by the control animals on the subsequent probe trials suggest, by this argument, that control, but not lesioned, animals learned the location of the hidden platform on the initial block of probe trials when the platform was invisible. If this were true, then Experiment 1 could be viewed as an inappropriate test of Rawlins's hypothesis. Although there is nothing in the data to preclude the above account, it cannot be generally true because there is substantial evidence that rats do learn the spatial locations of visible platforms (Morris, 1981; Schenk, 1985; Rudy \& Paylor, 1988). Nonetheless, the alternative account cannot be dismissed by our data. Thus, Experiment 2 was designed in part to provide a less ambiguous test of Rawlins's hypothesis. This was accomplished by using a working-memory version of the Morris (1981) place navigation task. Specifically, each day the platform was placed in a different location and the rat received four trials. Trials 1 and 3 of each session were with a visible platform, and Trials 2 and 4 were with the platform hidden.

\section{Method}

The apparatus and subjects were the same as those used in Experiment 1. During each of 10 training sessions, the platform was placed in one of four different locations. During the four-trial session, the platform remained in the same location. On Trials 1 and 3 of each session, the visible platform was placed in the pool; on Trials 2 and 4 , the hidden platform was placed in the pool. The subject was released from a different starting location on each of the four trials. The intertrial interval between trials with the visi- ble and hidden platform was $30 \mathrm{sec}$. The intertrial interval between Trials 2 and 3 was approximately $5 \mathrm{~min}$. During Session 11, the platform was placed in the NW quadrant of the pool, and the subjects were trained in the same manner as they had been on the previous 10 sessions except for one major difference: On Trial 4 of the session, the hidden platform was placed in the center of the diagonally opposite SE quadrant of the pool. Again, escape latencies and swim distance measures yielded the same outcome, and we will report only the escape-latency data.

\section{Results and Discussion}

The escape latencies for both controls and animals with damage to the hippocampal formation did not differ on visible-platform trials; they averaged less than $3 \mathrm{sec}$. Figure 3 presents the results of the trials with the hidden platform. Several points should be noted. First, the escape latencies on these trials were much shorter for the control animals than for the animals with damage to the hippocampal formation. Second, the latencies of both groups decreased between the first and second hiddenplatform trials of each session. These trends were confirmed statistically by a two-way repeated measures analysis of variance that revealed significant main effects of lesion $[F(1,11)=6.35, p<.05]$ and trials $[F(1,11)=$ 29.18, $p<.01]$. The lesion $\times$ trials interaction did not approach statistical significance $[F(1,1)=1.02]$, thereby confirming that the difference between the two groups was present on both the first and second probe trials of each session (see Figure 3).

The fact that the escape latencies of animals with damage to the hippocampal formation decreased between the first and second hidden platform trials of a session suggests that they might have learned something about the location of the platform. In this regard, it is instructive to consider their performance on the second hiddenplatform trial of Session 11. Recall that on that trial, we reversed the location of the platform from the NW to the

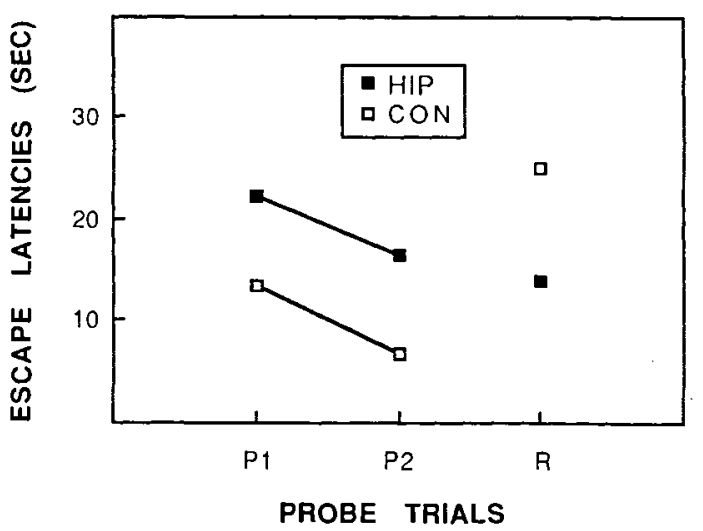

Figure 3. The mean escape latencies for the first (P1) and second (P2) prohe trial of each session averaged over the 10 training sessions. The point labeled $R$ represents the mean escape latencies of the animals on the second probe trial of Session 11 when the platform location was reversed. Note that on the reversal trial, the escape latency of the control group increased dramatically, whereas it decreased only slightly for the animals with hippocampal-formation damage. 
SE quadrant of the pool. If the decrease in second-trial latencies is truly reflective of the specific knowledge that the animal has acquired about the spatial location of the platform, and not due to some other nonspecific or motivational influence on performance, then reversing the platform location should actually result in increased swim latencies on this trial relative to the second-trial latencies obtained when the platform remained in the same location. Note that the control animals' behavior on the reversal trial suggests that they had knowledge of the platform's location. Their escape latencies increased dramatically relative to their normal second-trial performance-the latencies of 5 of the 6 rats increased when the platform location was reversed. In contrast, the animals with damage to the hippocampal formation displayed no such increase. Their latencies were, if anything, slightly reduced on the reversal trial relative to their normal second-trial performance-5 of the 7 animals escaped faster on the reversal trial than they did on their normal second trials. These findings suggest that the decrease in latency between the first and second hidden-platform trials displayed by the animals with hippocampal-formation damage reflects the influence of factors unrelated to specific location information.

These conclusions were supported by a two-way repeated measures analysis of variance that compared each subject's latency score on the second probe trial of Day 11 (when the location of the platform was reversed) with its second probe trial score averaged over Days 1-10 (when the platform remained in the same location). This analysis revealed only a lesion $\times$ trials interaction $[F(1,11)$ $=5.0, p<.05]$. An analysis of this interaction revealed that the control animals' performance differed $(p<.01)$ on the two trials, whereas the performance of the animals with damage to the hippocampal formation did not.

The importance of Experiment 1 for an evaluation of Rawlins's hypothesis was diminished by the fact that it provided no compelling evidence that control subjects acquired information about the location of the platform on trials with the visible platform in the pool. The finding that control animals differed from lesioned animals on the first probe trial of each session is important precisely because it provides this critical piece of evidence. Note that the platform's location varied from session to session and that the animal received only one trial with the visible platform prior to receiving the first probe trial. The fact that, on the first probe trial, the control animals located the platform significantly more quickly than did the hippocampal-damaged animals thus can only reflect knowledge about the platform's location that the control animals acquired by escaping to the visible platform. It is also important to note that the difference in escape latencies observed on the first probe trial cannot be attributed to any difference between the two groups on the first trial of each session with the visible platform. The mean Trial 1 escape latencies were 2.7 and $2.6 \mathrm{sec}$, respectively, for the hippocampal-damaged and control animals $(F<1)$.

\section{GENERAL DISCUSSION}

In two experiments, the temporal demands of the Morris (1981) place navigation task were reduced to a minimum by allowing rats to navigate to a visible platform in a fixed location. Nevertheless, animals with damage to the hippocampal formation were still significantly impaired relative to controls in their ability to find the hidden platform (Experiments 1 and 2) and displayed no evidence of knowing its location (Experiment 2). Thus, our experiments offer no support for Rawlins's $(1985,1987)$ theory that the hippocampal formation contributes to place learning in the Morris water task by enabling rats to span the temporal intervals typically observed with the hidden-platform version.

One might argue, however, that even though the lesioned animals' escape latencies averaged only $3 \mathrm{sec}$, this interval still exceeds the limits of what can be bridged in an animal without a hippocampal formation. In response to this argument, we note that the temporal limits associated with the temporary memory buffer theory have not been precisely specified, but it seems clear from the Rawlins (1987) and the Morris et al. (1986) papers that the short escape latencies associated with swimming to our visible platform $(<3 \mathrm{sec})$ should be well below an interval that requires the contribution of hypothetical "high-capacity, intermediate-term memory buffer" (Rawlins, 1985). For example, in extending Rawlins's argument to interpret their results, Morris et al. (1986) had to assume that 2-3 sec was within the temporal limits of the memory system of the rat with hippocampal damage.

The recent results of Okaichi (1987) also stand against Rawlins's view. Although he did not use the Morris water task, Okaichi's experiment was conceptually similar to Morris et al.'s (1986) cued-place discrimination task. Okaichi used an elevated T-maze to train animals to solve a pure place-learning task. The maze was rotated from trial to trial so that the goal, the end of the arm containing food, was always in the same spatial location, whereas the spatial location of the other arm varied. Moreover, this rotation sequence prevented the rat from solving the problem simply by learning to turn left or right at the choice point. Thus, the animal was faced with only two choices, and the structure of the maze channeled the animal's behavior so that the temporal demands of this task were minimal. Morris et al.'s (1986) explanation of why their animals with damage to the hippocampal formation were able to solve their cued-place discrimination would have to predict that Okaichi's animals would solve his place-learning task. Nevertheless, Okaichi's animals with hippocampal-formation damage never performed above chance. They were, however, readily able to solve a cued, nonspatial discrimination task.

The combination of Okaichi's (1987) results and our own leads us to suspect that Morris et al.'s (1986) rats with damage to the hippocampal formation learned the cuedplace discrimination task by discriminating between the 
fixed and floating platforms on the basis of some uncontrolled local cues within the pool rather than by truly discriminating on the basis of spatial locations.

\section{REFERENCES}

Morris, R. G. M. (1981). Spatial localization does not require the presence of local cues. Learning \& Motivation, 12, 239-260.

MorRs, R. G. M. (1982). An attempt to dissociate "spatial-mapping" and "working-memory" theories of hippocampal function. In W. Seifert (Ed.), Molecular, cellular and behavioural neurobiology of the hippocampus (pp. 405-432). New York: Academic Press.

Morris, R. G. M., Garrud, P., Rawlins, J. N. P., \& O'Keefe, J. (1982). Place navigation impaired in rats with hippocampal lesions. Nature, 297, 681-683.

Morris, R. G. M., Hagan, J. J., \& Rawlins, J. N. P. (1986)). Allocentric spatial learning by hippocampectomized rats: A further test of the "spatial mapping" and "working memory" theories of hippocampal function. Quarterly Journal of Experimental Psychology, 38B, 365-395.

OKaICHI, H. (1987). Performance and dominant strategies on place and cue tasks following hippocampal lesions in rats. Psychobiology, 15, 58-63.

O'KEEFE, J., \& NADEL, L. (1978). The hippocampus as a cognitive map. Oxford: Clarendon Press.

Rawlins, J. N. P. (1985). Associations across time: The hippocampus as a temporary memory store. Behavioral \& Brain Sciences, 8, $479-496$.
Rawuns, J. N. P. (1987). Time to close the store? Behavioral \& Brain Sciences, 10, 156-159.

Rudy, J. W., PAYLOR, R. (1988). Reducing the temporal demands of the Morris place-learning task fails to ameliorate the place-learning impairment of preweanling rats. Psychobiology, 16, 152-156.

Rudy, J. W., Stadler-Morris, S., \& Albert, P. (1987). Ontogeny of spatial navigation behaviors in the rat: Dissociation of "proximal-" and distal-cue based behaviors. Behavioral Neuroscience, 101, 62-73. SCHENK, F. (1985). Development of place navigation in rats from weaning to puberty. Behavioral \& Neural Biology, 43, 69-85.

SCHENK, F., \& MORRIS, R. G. M. (1985). Dissociation between components of spatial memory in rats after recovery from the effects of retrohippocampal lesions. Experimental Brain Research, 58, 11-28.

SUTHERLAND, R. J. (1985). The navigating hippocampus: An individual medley of space, memory and movement. In G. Buzsaki \& C. H. Vanderwolf (Eds.), Electrical activity of the archiconex (pp. 255-279). Budapest: Hungarian Academy of Sciences.

Sutherland, R. J., Kolb, B., \& WhiShaw, I. Q. (1982). Spatial mapping: Definite disruption by hippocampal or medial frontal cortical damage in the rat. Neuroscience Letters, 31, 271-276.

Sutherland, R. J., Whishaw, I. Q., \& Kolb, B. (1983). A behavioural analysis of spatial localization following electrolytic, kainate-, or colchicine-induced damage to the hippocampal formation in the rat. Behavioral Brain Research, 7, 133-153.

(Manuscript received October 4, 1987; revision accepted for publication February 26, 1988.) 